\title{
Traumatologie des Gesichtsschädels im Kindesalter
}

Christian Freudlsperger, Jürgen Hoffmann, Oliver Ristow

\section{Hintergrund}

Der Gesichtsschädel, das Viszerokranium, ist ein aus 15 Einzelknochen bestehender Teil des Schädels und umfasst das Mittelgesicht sowie den Unterkiefer (Mandibula). Er bildet damit die knöcherne Grundlage des Gesichts mit Augen- und Nasenhöhlen sowie der Mundhöhle. Frakturen des Gesichtsschädels im Kindealter sind einerseits für die betroffene Familie besonders belastend, stellen aber auch für den behandelnden Arzt oft eine große Herausforderung dar. Die hohen Erwartungen an die komplette Wiederherstellung der Strukturen des Gesichtsschädels und der damit verbundenen äußeren Erscheinung können zu einer anspruchsvollen Aufgabe werden. Der möglicherweise negative Einfluss der gewählten Therapie auf den sich im Wachstum befindlichen Knochen und damit auf die weitere Entwicklung erfordert ein höchstes Maß an Sorgfalt. Prinzipiell sollte die gewählte Therapie möglichst konservativ sein und wenn ein chirurgisches Vorgehen unvermeidbar ist, sollte dies so minimalinvasiv wie möglich sein. Die Fortschritte in Diagnostik und Therapie von Frakturen auf Mund-, Kiefer-, Gesichtschirurgischem Fachgebiet haben in den letzten Jahren dazu beigetragen, diese Aufgabe auf höchstem Niveau zu erfüllen. Trotzdem müssen wichtige Unterschiede beim sich im Wachstum befindlichen Knochen berücksichtigt werden, zumal der Großteil unseres heutigen Verständnisses auf der Versorgung von Frakturen des Gesichtsschädels bei der erwachsenen Bevölkerung beruht.

Dennoch bleiben die Grundprinzipien einer initialen umfassenden klinischen Untersuchung, einer korrekten Diagnose des Verletzungsmusters und ein individuell auf den Patienten abgestimmter Behandlungsplan bestehen.

\section{Wachstum und Entwicklung des Gesichtsschädels}

Viele der spezifischen Merkmale von Frakturen des Gesichtsschädels im Kindesalter lassen sich direkt auf die Unterentwicklung und auf das anhaltende Wachstum der Gesichtsschädelknochen zurückführen. Während Anteile des Kiefergelenks sich durch knorpelige Ossifikationsmechanismen (chondrale Ossifikation) entwickeln, entstehen die Knochen des Viszerokraniums über die membranöse (desmale) Ossifikation [1]. Hierbei ist das Knochenwachstum von einem Stimulus außerhalb des Knochens abhängig, wobei die Muskelaktivität eine wichtige Rolle spielt. Dieses Prinzip der von Melvin L. Moss beschriebenen funktionellen Matrix erklärt auch, warum Vernarbungen oder Kontrakturen des Weichgewebsmantels nach einem Trauma oder einem chirurgischen Eingriff verantwortlich für knöcherne Wachstumsstörungen sein können [2].

Einer der Hauptfaktoren, der die Inzidenz von kindlichen Gesichtsschädelfrakturen bedingt, ist das Verhältnis zwischen Hirnschädel- und Gesichtsschädelvolumen, das bei Geburt ungefähr 8:1 beträgt ( $\bullet$ Abb. 1 ). Dieser geringe Anteil des Mittelgesichts im Vergleich zum Kranium erklärt die höhere Inzidenz für Frakturen des Hirnschädels im Kindesalter [3,4]. Das anhaltende Wachstum des Gehirns expandiert das Kranium weiter, bis es im Alter von 5 Jahren bereits ungefähr 85\% der Größe eines Erwachsenen erreicht hat $[5,6]$. Im gleichen Zeitraum nimmt das Volumen der Augenhöhle deutlich zu und erreicht im Alter von 5 Jahren ca. 90\% seiner endgültigen Größe [7]. Das Mittelgesicht bleibt im Wachstum deutlich zurück und wächst sowohl in vertikaler und anteriorer Richtung, während das Nasenwachstum erst in späten Teenagerjahren seine angestrebte Erwachsenengröße erlangt [8]. Der Unterkiefer besitzt im Alter von 1 Jahr fast die Breite beim Erwachsenen, seine vollständige Höhe wird jedoch erst in den späten Teenagerjahren erreicht [8].

Der Grad der Pneumatisation der Nasennebenhöhlen wird für die geringere Häufigkeit von Mittelgesichtsfrakturen verantwortlich gemacht, da der Knochen massiver und damit stabiler ist. Die Nasennebenhöhlen wachsen in

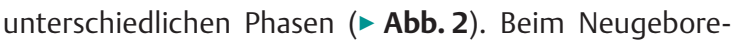
nen sind die Siebbeinzellen (Sinus ethmoidalis) bereits angelegt, während die restlichen Nasennebenhöhlen noch relativ unterentwickelt sind. Die Kieferhöhle (Sinus maxillaris) beginnt sich im 1. Lebensjahr zu entwickeln, zeigt aber erst im Alter von 5 Jahren ein deutliches Wachstum $[9,10]$. Die Stirnhöhle (Sinus frontalis) pneumatisiert am langsamsten mit einem Beginn im 2. Lebensjahr und kann teilweise erst im Alter von 8 Jahren radiologisch nachweisbar sein [11]. Erst im jungen Erwachsenalter erreicht die Stirnhöhle ihre vollständige Größe [12]. Zusätzlich bietet der Wangenfettkörper (Bichat- 


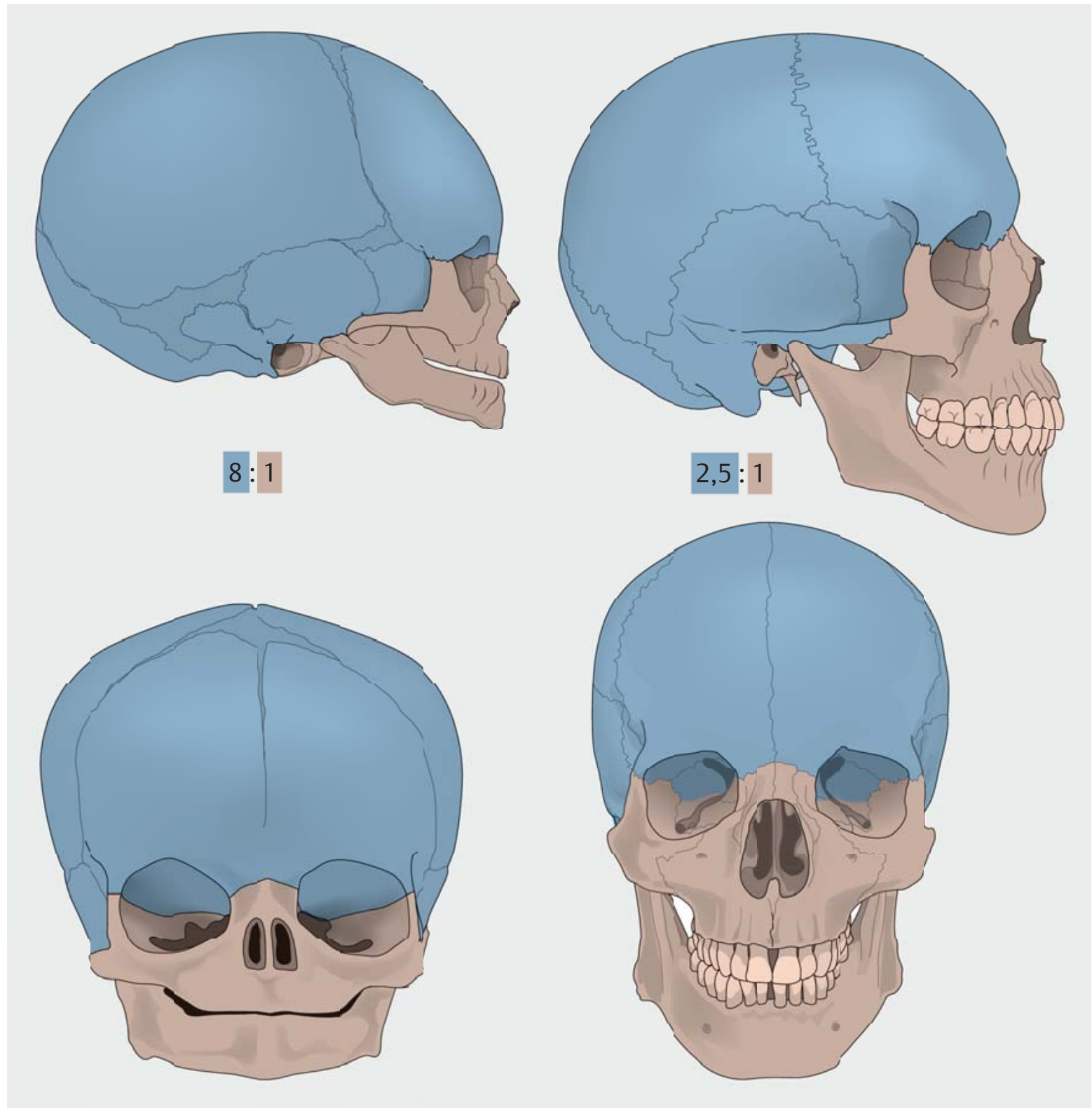

- Abb. 1 Gegenüberstellung eines Schädels eines Neugeborenen und eines Erwachsenen in der Ansicht von vorne und seitlich. Beim Neugeborenen beträgt das Verhältnis zwischen Hirnschädel- und Gesichtsschädelvolumen ungefähr 8: 1, beim Erwachsenen 2,5:1.

Fettkörper, Corpus adiposum buccae), der im Kindesalter noch deutlich ausgeprägter vorhanden ist, einen zusätzlichen Schutz gegenüber den von außen einwirkenden Kräften. Diese anatomischen Charakteristika des kindlichen Gesichtsschädels bedingen die unterschiedlichen Frakturmuster im Mittelgesichtsbereich im Gegensatz zu denen eines Erwachsenen. Während bei Kindern unter 2 Jahren vor allem die frontoorbitalen Strukturen betroffen sind, ähnelt das Frakturmuster beim Teenager bereits dem eines Erwachsenen [13].

\section{Epidemiologie}

Trotz großer Fortschritte im Kinderschutz bleibt ein Trauma die häufigste Ursache für kindliche Morbidität und Mortalität. Verletzungen im Gesichtsbereich sind in 11\% die Ursache für eine Vorstellung in einer pädiatrischen Notfallambulanz [1], wobei es sich hierbei in den meisten Fällen um Verletzungen der Zähne (dentoalveoläres Trauma) oder um reine Weichteilverletzungen handelt [14, 15]. 


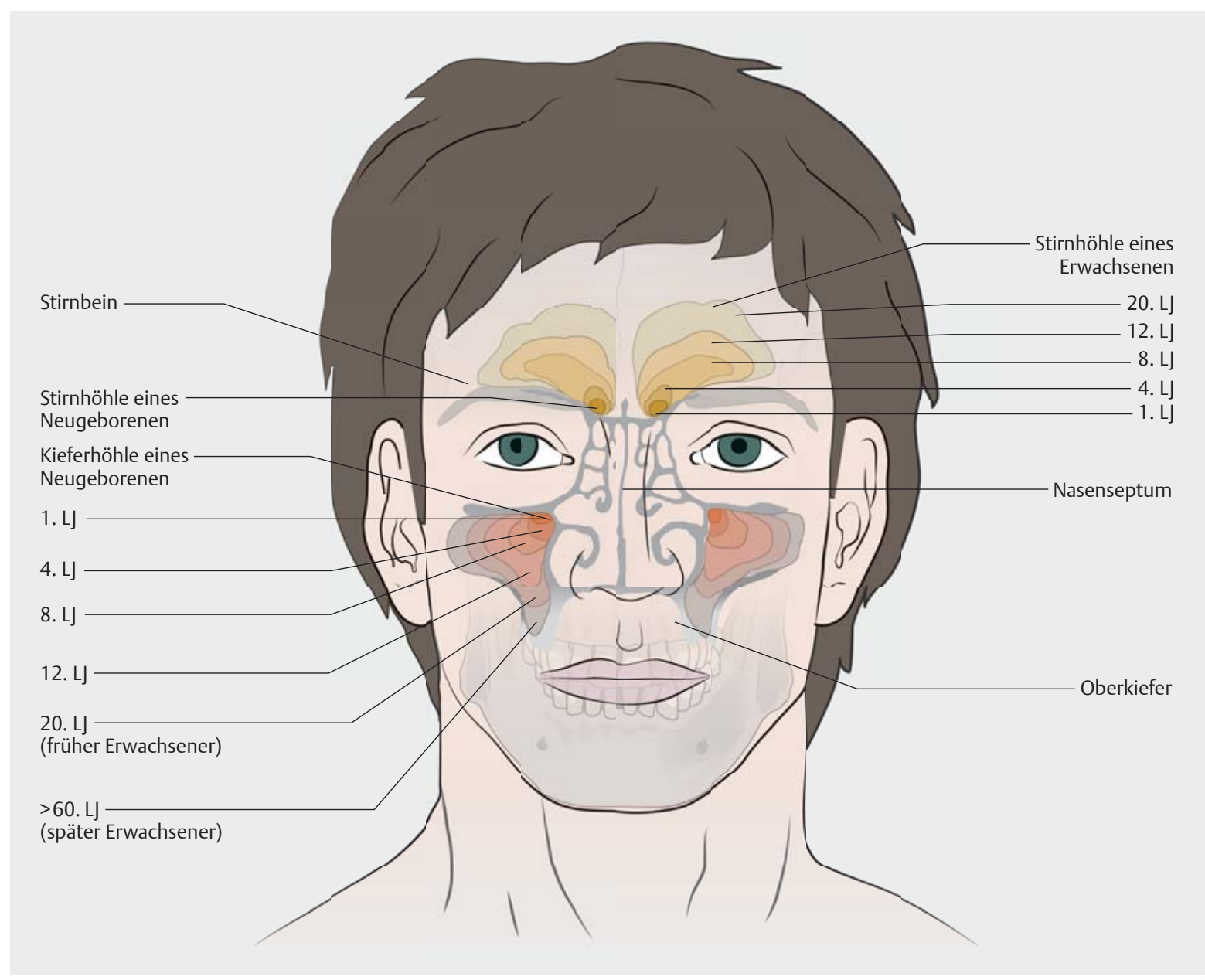

- Abb. 2 Entwicklung der Nasennebenhöhlen in Abhängigkeit vom Lebensalter.

Bezogen auf alle Patienten mit Frakturen des Gesichtsschädels machen Kinder jünger als 17 Jahre ungefähr 14,7\% aus [16], wobei ein Großteil dieser Patienten bereits im Teenageralter ist, während die Inzidenz bei Kindern jünger als 5 Jahre je nach Studie zwischen 1 und $5 \%$ liegt $[14,16]$. Die Häufigkeitsverteilung von Gesichtsschädelfrakturen in Abhängigkeit vom Alter zeigt 2 Höhepunkte: Der Erste im Alter von 6 bis 7 Jahren, der mit dem Beginn des Schulalters in Zusammenhang steht [17]. Der Zweite, mit 12 bis 14 Jahren, beruht auf der Zunahme an physischen Aktivitäten in diesem Lebensalter [18].

\section{Merke}

Bei einer Verletzung im Gesichtsbereich steigt das Risiko für eine Fraktur des Gesichtsschädels mit jedem Lebensjahr um $14 \%$.

Generell sind Jungen häufiger von Gesichtsschädelfrakturen betroffen als Mädchen, mit einer Relation, die je nach Studie von $1,1: 1$ bis zu 8,5:1 variiert $[19,20]$. In der Re- gel sind Stürze oder Sportunfälle ursächlich [21,22]. Die schwereren Frakturen treten oft im Rahmen von Verkehrsunfällen auf und sind die i.d.R. mit multiplen Begleitverletzungen verbunden [23]. Nicht unberücksichtigt dürfen Frakturen des Gesichtsschädels als Folge von Kindesmisshandlung bleiben [24]. In 2,3\% der misshandelten Kinder finden sich Frakturen im Gesichtsschädelbereich [17]

Die Häufigkeiten der Frakturlokalisationen. Imahara und Kollegen untersuchten anhand der Nationalen Traumadatenbank der Vereinigten Staaten insgesamt 277008 pädiatrische Traumapatienten und fanden bei 4,6\% der Patienten eine Fraktur des Gesichtsschädels [23]. 32,7\% der Frakturen waren im Bereich des Unterkiefers lokalisiert, 30,2\% betrafen die Nase und 28,6\% waren Frakturen im Bereich des Jochbeins und des Oberkiefers. In der Studie von Grunwaldt und Kollegen zeigten sich in der Altersgruppe jünger als 11 Jahre vor allem Frakturen der Augenhöhle und bei Kindern zwischen 12 und 18 Jahren hauptsächlich Frakturen des Unterkiefers [13]. 


\section{Diagnostik und bildgebende Verfahren}

Wie bei jedem Traumapatienten muss nach der primären Stabilisierung zunächst der Fokus auf der Diagnose und Versorgung von lebensbedrohlichen Verletzungen gelegt werden, bevor Verletzungen im Gesichtsbereich behandelt werden. Aufgrund des im Kindesalter relativ kleinen Gesichtsschädels und der hohen Dichte des Knochens stellen Frakturen in diesem Bereich häufig ein Indiz auf ein Hochrasanztrauma dar [13].

Merke

Cave: Bei 55\% der kindlichen Gesichtsschädelfrakturen liegen weitere Begleitverletzungen vor.

Ein besonderes Augenmerk muss hierbei auf mögliche intrakranielle Verletzungen gelegt werden, die bei ungefähr 57\% der Kinder unter 5 Jahren mit einer Fraktur des Gesichtsschädels vorliegen [23]. Im Gegensatz zu Erwachsenen, bei denen in ungefähr $10 \%$ der Fälle eine zusätzliche Verletzung der Halswirbelsäule vorliegt, sind diese Begleitverletzungen im Kindesalter eher seltener anzutreffen $(0,9-2,3 \%)[25,26]$.

Bei Frakturen mit Beteiligung der Augenhöhle ist eine augenärztliche Untersuchung unerlässlich, um eine Verletzung des Auges auszuschließen, was bei ca. 50\% der Fälle auftritt [13]. In 0,5-0,3\% der Augenverletzungen droht eine Erblindung [27]. Die Untersuchung der Augen sollte die Pupillomotorik, den Visus, Doppelbilder und die Augenbeweglichkeit miteinschließen, soweit dies in Abhängigkeit vom Alter möglich ist.

Eine umfassende klinische Untersuchung ist i.d. R. durch die mangelnde Kooperation des Kindes erschwert. Der knöcherne Rand der Augenhöhle kann auf Knochenstufen palpiert werden, die Beurteilung der Kontur der Jochbögen ist jedoch aufgrund des bei Kindern erhöhten Fettgewebes in diesem Bereich oft nicht möglich.

Bei klinischem Verdacht auf eine Fraktur des Gesichtsschädels sind bildgebende Verfahren essenziell, zum einen für die Diagnosesicherung, aber auch für die Wahl der optimalen Therapie. Für eine initiale Bildgebung kann eine Ultraschalluntersuchung hilfreich sein und falls ein Frakturverdacht vorliegt, können weiter gezielte röntgenologische Aufnahmen veranlasst werden. Übersichtsröntgenaufnahmen sind im Kindesalter bekanntermaßen unzuverlässig: Aufgrund der unterentwickelten Nasennebenhöhlen, der nicht durchgebrochenen Zähne und der Neigung zu Grünholzfrakturen stellen sich Frakturen des Gesichtsschädels oft nur sehr gering oder überhaupt nicht disloziert da [28].

Zusätzlich erschwert die oft fehlende Kooperation des Kindes eine exakte Positionierung, die für bestimmte Schädelprojektionen notwendig ist [28]. Die Computertomografie hat, soweit verfügbar, in der Diagnostik von kindlichen Gesichtsschädelfrakturen insbesondere im Mittelgesichtsbereich, die konventionellen Schädelaufnahmen weitestgehend ersetzt.

Merke

Der Goldstandard zur Diagnostik von Gesichtsschädelfrakturen im Kindesalter bleibt die Computertomografie (CT).

Insbesondere die koronaren und sagittalen Schichten einer CT-Untersuchung erlauben eine exakte Darstellung von Frakturen im Bereich der Augenhöhlen und des Mittelgesichts und erhöhen die diagnostische Sicherheit [29]. Darüber hinaus bietet eine 3-dimensionale Rekonstruktion eine exakte Darstellung der anatomischen Strukturen und ist insbesondere zur Planung einer chirurgischen Versorgung äußerst hilfreich. Generell sollte die Verwendung von ionisierenden Strahlen gerade bei Kindern auf ein Minimum reduziert werden und muss, falls appliziert, einen definitiven Nutzen für den Patienten haben. In der Mund-, Kiefer-, Gesichtschirurgie wird als schichtbildgebendes Verfahren in den letzten Jahren die digitale Volumentomografie (DVT) favorisiert, die eine exakte Frakturdiagnostik und Planung der Therapie bei im Vergleich zur konventionellen CT-Untersuchung deutlich reduzierter Strahlenbelastung erlaubt. Zur Diagnostik von Unterkieferfrakturen eignet sich nach wie vor die Panoramaschichtaufnahme (Orthopantomogramm). Die Panoramaschichtaufnahme gewährleistet eine zuverlässige Diagnose einer Fraktur des Unterkieferkorpus und ist sowohl für die Planung einer operativen Versorgung sowie für die postoperative Stellungskontrolle unerlässlich. Zur Darstellung der 2. Ebene werden i. d. R. p.-a. 15-Aufnahmen vom Schädel (Clementschitsch-Aufnahme) verwendet.

\section{Mittelgesichtsfrakturen}

Die Einteilung der Mittelgesichtsfrakturen unter funktionell-topografischen Gesichtspunkten hat sich in den letzten Jahren zunehmend durchgesetzt: Zentrale Mittelgesichtsfrakturen, laterale Mittelgesichtsfrakturen und die Kombination beider Formen im Sinne von zentrolateralen Mittelgesichtsfrakturen. 


\section{INFOBOX 1}

\section{Einteilung der Mittelgesichtsfrakturen}

Zentrale Mittelgesichtsfrakturen

- Le-Fort-Frakturen (I und II)

- Nasenskelettfrakturen

- Naso-Orbito-Ethmoidale Frakturen (NOE-Frakturen)

\section{Laterale Mittelgesichtsfrakturen}

- Zygomatikoorbitale Frakturen

- Orbitabodenfrakturen

- Jochbogenfrakturen

\section{Zentrolaterale Mittelgesichtsfrakturen}

- Le-Fort-III-Fraktur

Zu den zentralen Mittelgesichtsfrakturen zählen Frakturen mit basaler Absprengungen des Oberkiefers (LeFort-I-Frakturen), mit vollständigem Abriss des Mittelgesichts von der Schädelbasis (Le-Fort-II-Fraktur) und Frakturen des Nasenskeletts (Nasomaxilläre und nasoorbitoethmoidale Frakturen). Aufgrund der bereits beschriebenen anatomischen Besonderheiten des kindlichen Gesichtsschädels sind Frakturen gemäß der klassischen Le-Fort-Einteilung relativ selten und stehen, falls vorhanden, oft mit einem Hochrasanztrauma in Zusammenhang [30]. Mit der Entwicklung der Nasennebenhöhlen, dem Durchbruch der bleibenden Zähne und dem Wachstum des Gesichtsschädels nimmt auch die Inzidenz der zentralen Mittelgesichtsfrakturen zu [17].

Das knöcherne Nasenskelett ist der am wenigsten widerstandsfähige Knochen des Gesichtsschädels, und Frakturen des Nasenskeletts ( $\bullet$ Abb. $\mathbf{3}$ ) zählen zu den häufigsten Frakturen im Kindesalter, zumal ihre wahre Inzidenz in der Literatur sicherlich unterrepräsentiert ist [31]. Dies ist mit der Tatsache verbunden, dass die meisten Nasenskelettfrakturen isoliert auftreten ohne weitere Begleitverletzungen und aus diesem Grund eher im ambulanten Bereich behandelt werden. Zusätzlich erschwert die anfängliche Weichteilschwellung die Beurteilung der Symmetrie des Nasenbeins, sodass viele Frakturen initial nicht diagnostiziert werden. Bei Verdacht auf eine Nasenskelettfraktur kann ein seitliches Röntgenbild die Diagnose bestätigen ( $\mathbf{A} \mathbf{b b}$. 3). Zum Ausschluss eines Septumhämatoms, wenn auch selten, sollte immer eine vordere Nasenspiegelung (Rhinoskopie) durchgeführt werden. Beim Vorliegen eines Septumhämatoms muss eine sofortige Entlastung erfolgen, um eine Nekrose des Knorpels mit möglicher Sattelnase zu vermeiden.

Innerhalb der ersten Tage nach dem Trauma sollte die Versorgung einer Nasenskelettfraktur durch geschlossene Reposition erfolgen [32,33]. Im Gegensatz zu reinen

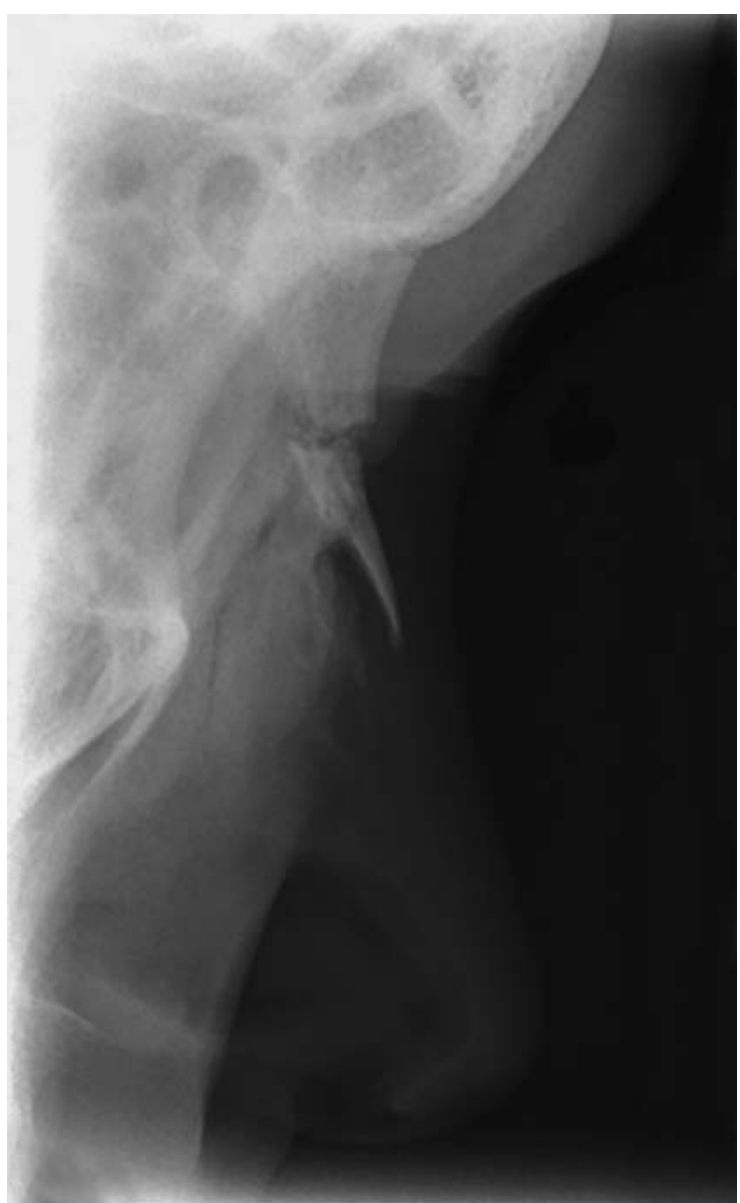

- Abb. 3 Röntgenologische Darstellung einer Nasenskelettfraktur eines 11-jährigen Patienten. Aufnahmetechnik: Nasenbein seitlich.

Nasenskelettfrakturen sind Nasoorbitoethmoidale (NOE) Frakturen relativ selten im Kindesalter [28,34]. Aufgrund des i. d. R. relativ flachen Nasenrückens bei Kindern ist die Unterscheidung einer NOE-Fraktur von einer einfachen Nasenskelettfraktur mit einer klinischen Untersuchung oft schwierig. Ein in das Mittelgesicht impaktierter Nasenrücken (Sattelnase) oder ein Telekanthus können hier richtungsweisend sein, oft wird jedoch eine CT-Diagnostik benötigt.

\section{Merke}

Eine Sattelnase oder ein Telekanthus kann ein Hinweis auf eine nasoorbitoethmoidale (NOE) Fraktur sein.

Die Versorgung einer NOE-Fraktur erfolgt i.d.R. primär chirurgisch über eine offene Reposition mit interner Fixation, entweder über eine bereits vorhandene Verletzung im Bereich der Augenbraue oder über einen bikoronaren Zugang.

Die lateralen Mittelgesichtsfrakturen erstrecken sich auf Jochbein, Jochbogen, Orbita und Oberkiefer. Häufig zu 

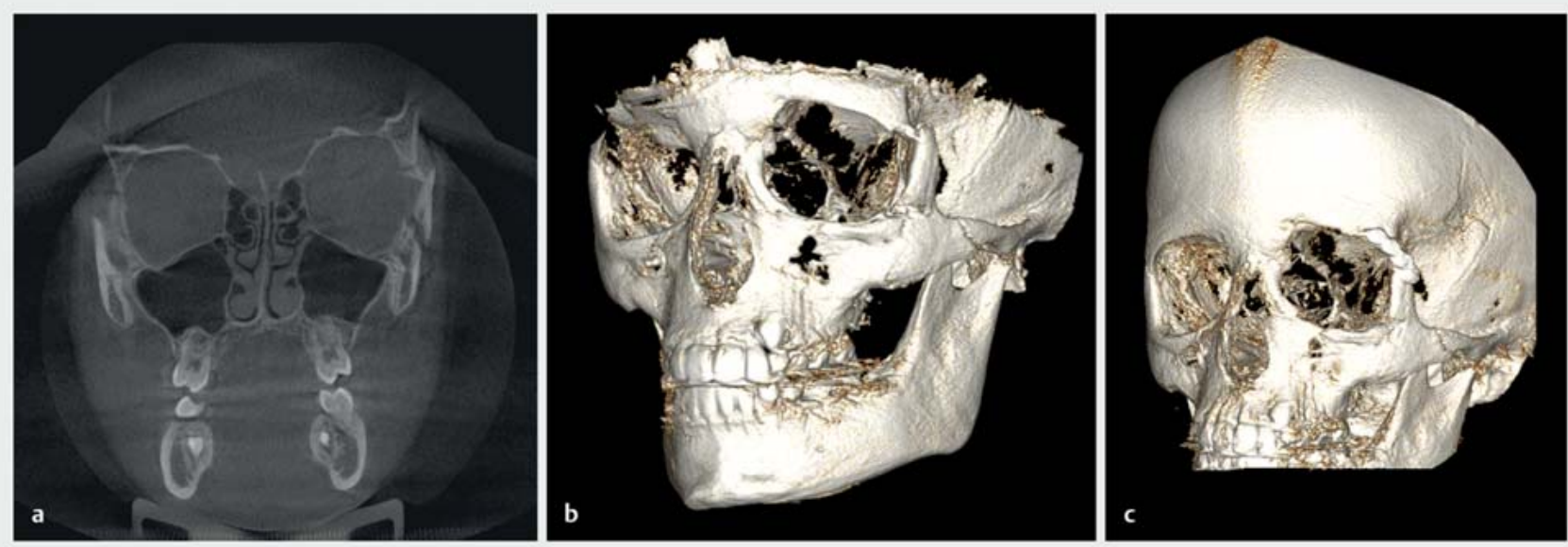

- Abb. 4 Röntgenologischer Nachweis einer Fraktur des zygomatikoorbitalen Komplexes links bei einem 12-jährigen Patienten mittels digitaler Volumentomografie. a Koronare Schichtung präoperativ. b Dreidimensionale Rekonstruktion präoperativ. c Dreidimensionale Rekonstruktion postoperativ nach interner Fixierung mittels Miniplattenosteosynthese.

beobachtende Frakturformen sind: Frakturen des zygomatikoorbitalen Komplexes und Frakturen des Orbitabodens.

Frakturen des zygomatikoorbitalen Komplexes zählen mit 7-41\% zu den häufigsten Frakturen im Mittelgesichtsbereich $[17,18]$ ( $\triangleright$ Abb.4a,b). Eine Vielzahl der Frakturen zeigt nur eine geringfügige Dislokation, sie können konservativ behandelt werden. Frakturen mit einer deutlichen Dislokation sollten jedoch über eine offene Reposition und interne Fixierung versorgt werden ( $\bullet \mathbf{A b b} . \mathbf{4} \mathbf{c}$ ). Aufgrund einer möglichen Wachstumshemmung empfehlen viele Autoren eine Entfernung des Osteosynthesematerials nach 3-4 Monaten [35].

Frakturen des Orbitabodens sind ab einem Alter von 5 Jahren häufiger als Frakturen des Orbitadaches [13]. Der übliche Frakturmechanismus einer Orbitabodenfraktur besteht in einem stumpfen Trauma auf den Augapfel, was zu seiner Dorsalbewegung führt. Dadurch werden die orbitalen Weichteile komprimiert und der intraorbitale Druck steigt an. Die Orbitahöhle gibt an ihrer schwächsten Stelle, dem Orbitaboden, nach und bricht trichterförmig in die Kieferhöhlen ein. In Analogie zum Bruchmechanismus wurde der Begriff der „Blow-out“Fraktur eingeführt.

\section{Merke}

Der häufigste Manifestation einer Orbitabodenfraktur ist die „Blow-out“-Fraktur.

Klassische klinische Zeichen einer Orbitabodenfraktur sind ein Enophthalmus aufgrund des vergrößerten orbitalen Volumens und das Vorhandensein von Doppelbildern, die vor allem beim Blick nach oben auftreten können [36].
Aufgrund der Neigung zu Grünholzfrakturen besteht bei Frakturen des Orbitabodens im Kindesalter die Gefahr einer sog. „Trap-Door“-Fraktur, bei der es zu einer Einklemmung eines äußeren Augenmuskels (M. rectus inferior) mit entsprechender Einschränkung der Augenbeweglichkeit kommen kann ( $\bullet$ Abb. 5). Die Symptome der akuten Einklemmung können sehr irreführend sein und eine Contusio cerebri imitieren. Die Patienten klagen über Schmerzen, Übelkeit, Erbrechen, Bradykardien und Synkopen (okulokardialer Reflex) [37] In diesen Fällen ist die sofortige chirurgische Intervention zur Entlastung des eingeklemmten Gewebes sehr dringlich, um eine irreversible Muskelschädigung mit der Konsequenz einer Vernarbung und permanenten Doppelbildwahrnehmung zu vermeiden.

Merke

Eine absolute Indikation zur operativen Versorgung im Sinne eines pädiatrischen Notfalls ist bei einer Orbitabodenfraktur mit Muskeleinklemmung gegeben („Trap-Door“-Fraktur).

Für die chirurgische Versorgung einer Orbitabodenfraktur können unterschiedliche operative Zugangswege gewählt werden, wobei der transkonjunktivale Zugang aus ästhetischer Sicht favorisiert wird, der zusätzlich die postoperative Komplikation eines Ektropiums reduziert [38].

\section{Unterkieferfrakturen}

Frakturen des Unterkiefers (Mandibula) im Kindesalter unterscheiden sich insbesondere von denen bei Erwachsenen durch das Vorhandensein von nicht durchgebrochenen Zähnen oder Zahnkeimen im Unterkieferknochen. Die noch nicht durchgebrochenen Zähne und 

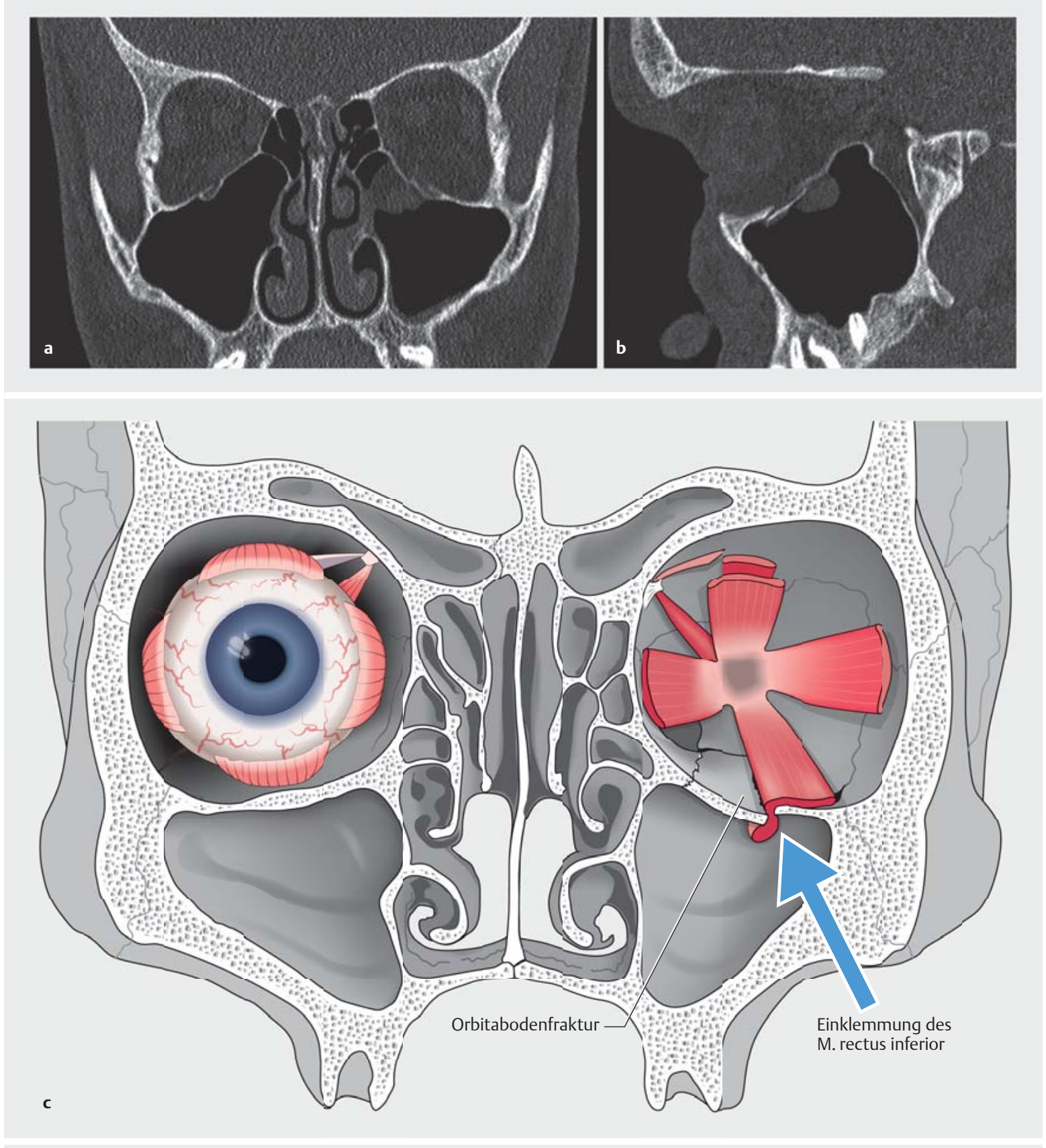

- Abb. 5 Computertomografischer Nachweis einer Orbitabodenfraktur links bei einem 14-jährigen Patienten mit Einklemmung des M. rectus inferior („Trap-Door“-Fraktur). a Koronare Schichtung. b Sagittale Schichtung. c Schematische Darstellung des Frakturmechanismus einer „Trap-Door“-Fraktur.

Zahnkeime tragen zu einer höheren Dichte des Knochens und damit zu einer höheren Stabilität bei [21].

Frakturen des Unterkiefers gehören mit Sicherheit zu den häufigsten Frakturen des Gesichtsschädels im Kindesalter [19]. Bei Kindern liegt i.d. R. eine Einfachfraktur des Unterkiefers vor, im Gegensatz zu Erwachsenen, bei denen der Unterkiefer meistens an 2 oder mehreren Stellen bricht. Am häufigsten ist der Gelenkfortsatz (Processus condylaris) betroffen, wobei viele Frakturen in dieser Lo- kalisation wahrscheinlich undiagnostiziert bleiben [39]. Gelenkfortsatzfrakturen treten meist beidseitig auf und verlaufen bei Kindern jünger als 6 Jahre meist intrakapsulär im Bereich des Gelenkköpfchens (Caput mandibulae) ( $\bullet$ Abb. 6). Bei älteren Kindern sind Gelenkfortsatzfrakturen meist im Bereich des Unterkieferhalses (Collum mandibulae) zu finden.

Frakturen im Bereiche der Unterkiefersymphyse sind am zweithäufigsten im Kindesalter. Im jugendlichen Alter 


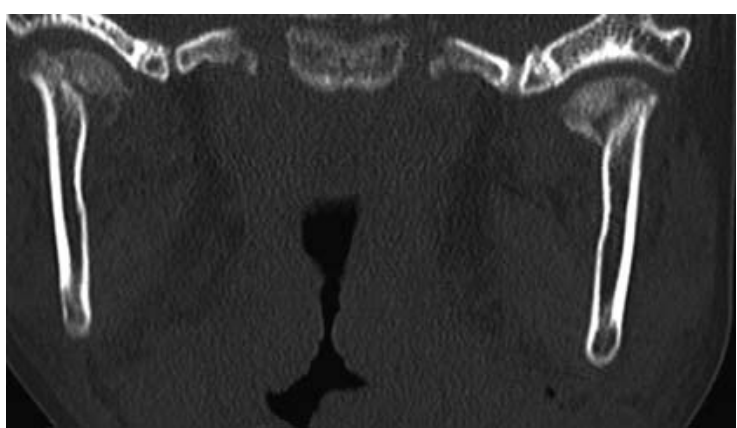

- Abb. 6 Nachweis einer intrakapsulären Fraktur des Kiefergelenkköpfchens beidseits mittels Computertomografie in koronarer Schichtung bei einem 7-jährigen Patienten.

nehmen Frakturen im Bereich des Unterkieferkorpus und im Kieferwinkel zu [40].

Bei der chirurgischen Versorgung von kindlichen Unterkieferfrakturen müssen bestimmte Besonderheiten berücksichtigt werden: noch nicht durchgebrochene Zähne und Zahnkeime dürfen nicht verletzt werden und mögliche spätere Wachstumsstörungen müssen vermieden werden. Bei der Wahl der Versorgungsmöglichkeit muss der Behandler von daher die Frakturlokalisation, die aktuelle Wachstumsphase des Kieferknochens sowie die Zahnentwicklung berücksichtigen.

\begin{abstract}
Merke
Im Kindesalter können viele Unterkieferfrakturen rein konservativ behandelt werden.
\end{abstract}

Bei sehr jungen Kindern können nicht dislozierte Frakturen, die keine Auswirkung auf die Okklusion zeigen, konservativ allein durch weiche Kost therapiert werden. Als zusätzliche Maßnahme kann im bezahnten Kiefer eine Ruhigstellung mittels mandibulomaxillärer Fixierung über an den Kiefern angebrachten Schienen erfolgen, wobei dies nur über einen kurzen Zeitraum von 7-10 Tagen empfohlen wird, um eine Ankylose im Kiefergelenk zu vermeiden [40,41] ( Abb. 7). Bei dislozierten Frakturen oder bei gestörter Okklusion kann mit offener Reposition und interner Fixierung versorgt werden, wobei hier bei der Platzierung des Osteosynthesematerials noch nicht durchgebrochene Zähne und Zahnkeime berücksichtigt werden müssen ( $\boldsymbol{A} \mathbf{A b b} \mathbf{b}$ ). In der Regel ist eine einzelne Miniplatte, die am Unterkieferbasalrand mit monokortikalen Schrauben fixiert wird, ausreichend ( $\triangleright$ Abb. 8). Nicht unberücksichtigt darf dabei die Tatsache bleiben, dass für eine interne Fixierung ein chirurgischer Zugang sowie eine subperiostale Präparation erforderlich ist, bei der das Periost verletzt und dadurch spätere Wachstumsstörungen verursacht werden können.

Dennoch bleiben Wachstumsstörungen nach Unterkieferfrakturen im Kindesalter ein großes Problem. Häufig sind Frakturen im Gelenkfortsatz ursächlich für spätere
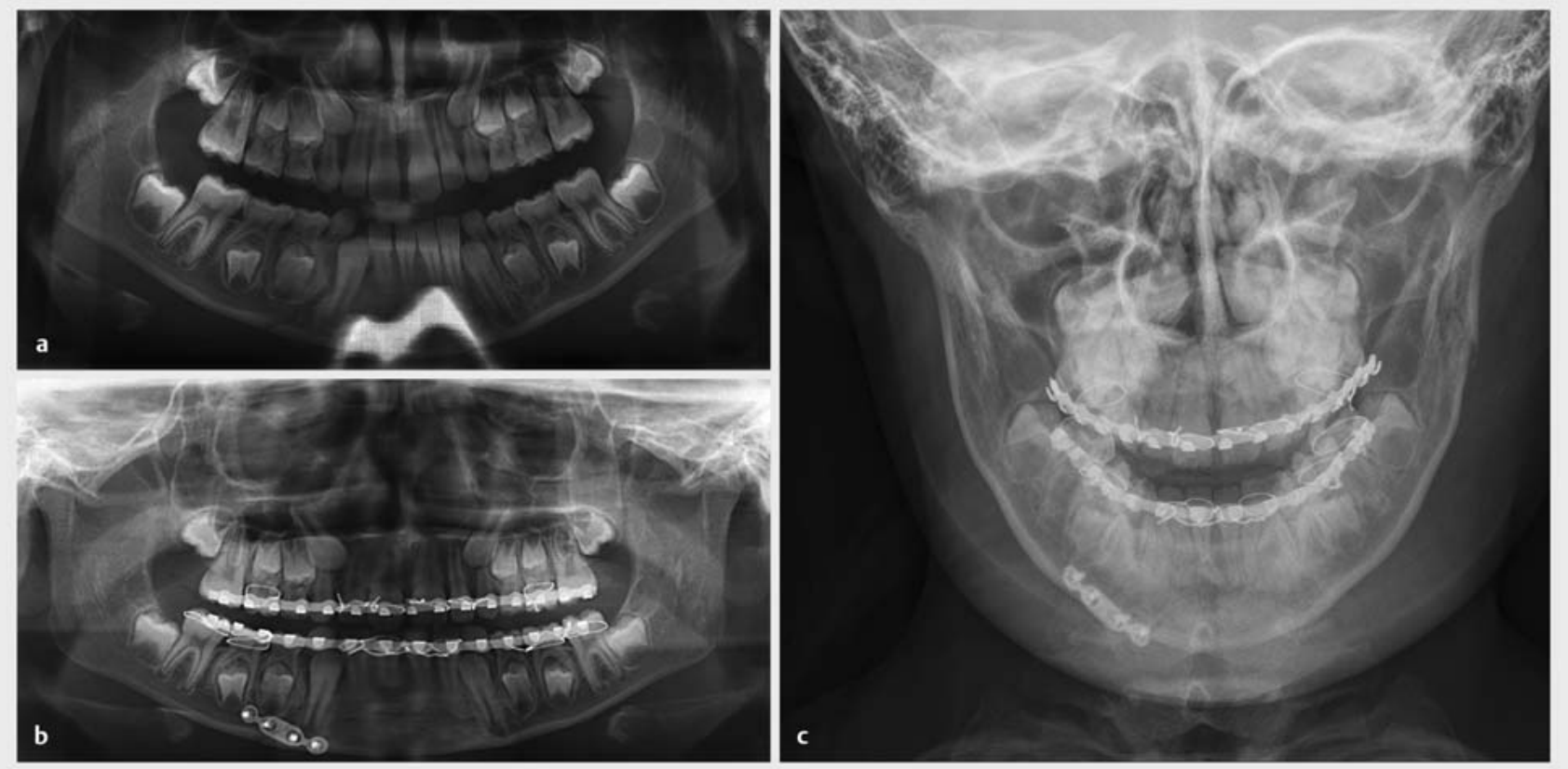

- Abb. 7 Darstellung einer Unterkiefer-2-fach-Fraktur paramedian rechts und tiefe Gelenkfortsatzfraktur links bei einer 9-jährigen Patientin. a Präoperative Panoramaschichtaufnahme. b Postoperative Panoramaschichtaufnahme mit interner Fixierung der paramedianen Fraktur mittels Miniplattenosteosynthese und konservativer Versorgung der Gelenkfortsatzfraktur links mittels Schienung und mandibulomaxillärer Fixierung. c Postoperative p.-a. $15^{\circ}$-Aufnahme des Schädels (Clementschitsch-Aufnahme) zur Darstellung der 2. Ebene. 

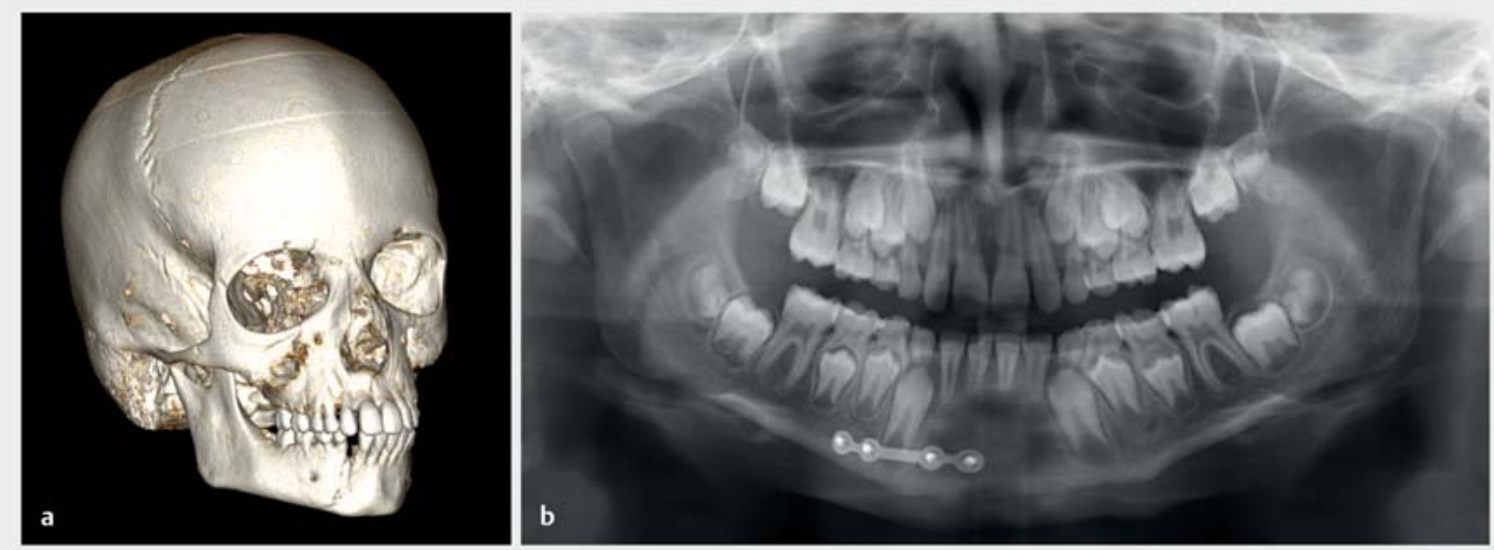

- Abb. 8 Röntgenologische Darstellung einer Unterkieferfraktur paramedian rechts im Bereich der Korpusregion bei einem 11jährigen Patienten. a Präoperative 3-dimensionale Darstellung. b Postoperative Röntgenkontrolle mit Panoramaschichtaufnahme nach interner Fixierung mittels Miniplattenosteosynthese.

Wachstumsstörungen des Unterkiefers, da dieser Bereich das primäre Wachstumszentrum darstellt [42] ( $\bullet$ Abb. 9). Demianczuk und Kollegen konnten zeigen, dass in $24 \%$ der Fälle einer Gelenkfortsatzfraktur in der Phase des ver- tikalen Wachstums im Erwachsenalter eine Kieferfehlstellung vorliegt, bei der eine Kieferumstellungsoperation benötigt wird [41]. Eltern sollten frühzeitig über diese möglichen Spätfolgen informiert werden, um entspre-
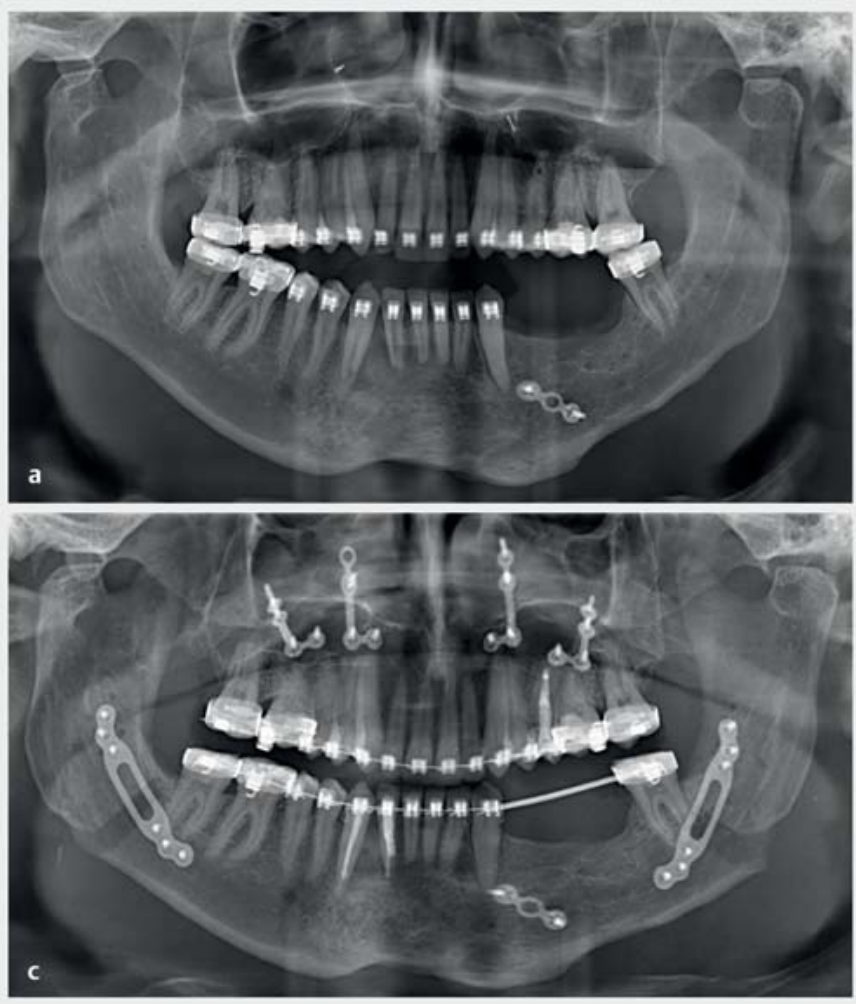
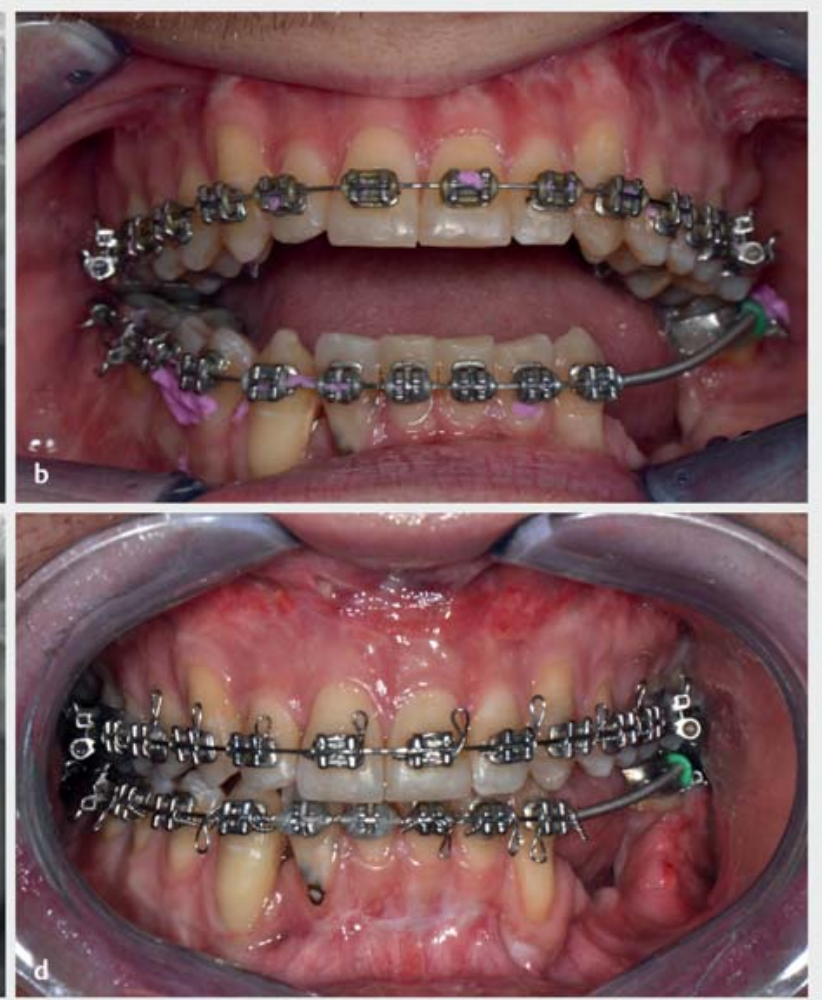

- Abb. 9 Darstellung einer Kieferfehlstellung in Form eines frontal offenen Bisses als Folge einer Unterkiefer-3-fach-Fraktur: Gelenkfortsatz beidseits und Korpusbereich links. a Präoperative Panoramaschichtaufnahme mit noch einliegendem Osteosynthesematerial, Unterkieferkorpus links. b Präoperativer intraoraler Befund mit frontal offenem Biss und Zahnkontakt ausschließlich im Molarenbereich unter laufender kieferorthopädischer Behandlung. c Postoperative Panoramaschichtaufnahme nach bignather Umstellungsosteotomie und einliegendem Osteosynthesematerial. d Postoperativer intraoraler Befund mit regelrechter Okklusion. 
chende kieferorthopädische Behandlungsmaßnahmen rechtzeitig einzuleiten.

\section{INFOBOX 2}

\section{Resorbierbare Osteosynthesematerialien}

Ein weiterhin viel diskutiertes Thema ist die Verwendung von resorbierbarem Osteosynthesematerial bei der Versorgung kindlicher Gesichtsschädelfrakturen. Resorbierbare Osteosynthesematerialien finden bereits einen weit verbreiteten Einsatz in der kraniofazialen Chirurgie und besitzen gegenüber konventionellen Titanosteosyntheseplatten den Vorteil, dass der zur Entfernung der Platten erforderliche Zweiteingriff entfällt [43]. Die früheren Nachteile von resorbierbaren Materialien wie geringere Stabilität trotz breiterem Durchmesser und möglichen Entzündungsreaktionen beim Auflösen der Platten sind mittlerweile durch die Entwicklung neuerer Produkte überwunden. In Bereichen, wo die Platte unmittelbar unter der Haut zu liegen kommt, kann durch das Aufquellen der Platte im Rahmen des Auflösungsprozesses eine vorübergehende Schwellung auftreten [43]. Dennoch lässt die aktuelle Datenlage zum heutigen Zeitpunkt keine eindeutige Empfehlung für resorbierbares Osteosynthesematerial in der Versorgung von kindlichen Frakturen des Gesichtsschädels zu.
Autorinnen/Autoren
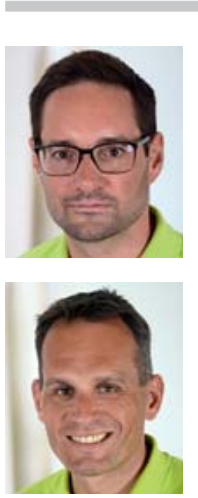

\section{Christian Freudlsperger}

Prof. Dr. Dr., Oberarzt, Klinik und Poliklinik für Mund-, Kiefer- und Gesichtschirurgie, Universitätsklinikum Heidelberg

\section{Jürgen Hoffmann}

Prof. Dr. Dr., Ärztlicher Direktor, Klinik und Poliklinik für Mund-, Kiefer- und Gesichtschirurgie, Universitätsklinikum Heidelberg

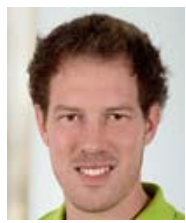

\section{Oliver Ristow}

Dr. Dr., Facharzt, Klinik und Poliklinik für Mund-, Kiefer- und Gesichtschirurgie, Universitätsklinikum Heidelberg

Korrespondenzadresse

Prof. Dr. Dr. Christian Freudlsperger

Klinik und Poliklinik für Mund-, Kiefer- und Gesichtschirurgie Universitätsklinikum Heidelberg

Im Neuenheimer Feld 400

69120 Heidelberg

Tel.: $06221 / 5638318$

Fax: $06221 / 564222$

Chr.freudlsperger@med.uni-heidelberg.de

\section{Literatur}

[1] Morris C, Kushner GM, Tiwana PS. Facial skeletal trauma in the growing patient. Oral Maxillofac Surg Clin North Am 2012; 24 : 351-364

[2] Moss ML. The functional matrix hypothesis revisited. 1. The role of mechanotransduction. Am J Orthod Dentofacial Orthop 1997; 112: 8-11

[3] Cole P, Kaufman Y, Hollier LH. Managing the pediatric facial fracture. Craniomaxillofac Trauma Reconstr 2009; 2: 77-83

[4] Gussack GS, Luterman A, Powell RW et al. Pediatric maxillofacial trauma: unique features in diagnosis and treatment. Laryngoscope 1987; 97: 925-930

[5] Sgouros S, Goldin JH, Hockley AD et al. Intracranial volume change in childhood. J Neurosurg 1999; 91: 610-616

[6] Farkas LG, Posnick JC, Hreczko TM. Anthropometric growth study of the head. Cleft Palate Craniofac J 1992; 29: 303-308

[7] Farkas LG, Posnick JC, Hreczko TM et al. Growth patterns in the orbital region: a morphometric study. Cleft Palate Craniofac J 1992; 29: 315-318

[8] Farkas LG, Posnick JC, HreczkoTM. Growth patterns of the face: a morphometric study. Cleft Palate Craniofac J 1992; 29: 308315

[9] Kao J, Sikora AT, Fu S. Dual EGFR and COX-2 inhibition as a novel approach to targeting head and neck squamous cell carcinoma. Curr Cancer Drug Targets 2009; 9: 931-937

[10] Wolf G, Anderhuber W, Kuhn F. Development of the paranasal sinuses in children: implications for paranasal sinus surgery. Ann Otol Rhinol Laryngol 1993; 102: 705-711 
[11] Yavuzer R, Sari A, Kelly CP et al. Management of frontal sinus fractures. Plast Reconstr Surg 2005; 115: 79e-93e

[12] Brown WA, Molleson TI, Chinn S. Enlargement of the frontal sinus. Ann Hum Biol 1984; 11: 221-226

[13] Grunwaldt L, Smith DM, Zuckerbraun NS et al. Pediatric facial fractures: demographics, injury patterns, and associated injuries in 772 consecutive patients. Plast Reconstr Surg 2011; 128: $1263-1271$

[14] Gassner R, Tuli T, Hächl O et al. Cranio-maxillofacial trauma: a 10 year review of 9,543 cases with 21,067 injuries. J Craniomaxillofac Surg 2003; 31: 51-61

[15] Alvi A, Doherty T, Lewen G. Facial fractures and concomitant injuries in trauma patients. Laryngoscope 2003; 113: 102-106

[16] Vyas RM, Dickinson BP, Wasson KL et al. Pediatric facial fractures: current national incidence, distribution, and health care resource use. J Craniofac Surg 2008; 19: 339-349

[17] Zimmermann CE, Troulis M], Kaban LB. Pediatric facial fractures: recent advances in prevention, diagnosis and management. Int J Oral Maxillofac Surg 2006; 35: 2-13

[18] Haug RH, Foss J. Maxillofacial injuries in the pediatric patient. Oral Surg Oral Med Oral Pathol Oral Radiol Endod 2000; 90: 126-134

[19] Ferreira PC, Amarante JM, Silva PN et al. Retrospective study of 1251 maxillofacial fractures in children and adolescents. Plast Reconstr Surg 2005; 115: 1500-1508

[20] Holland A], Broome C, Steinberg A et al. Facial fractures in children. Pediatr Emerg Care 2001; 17: 157-160

[21] Gassner R, Tuli T, Hächl O et al. Craniomaxillofacial trauma in children: a review of 3,385 cases with 6,060 injuries in 10 years. J Oral Maxillofac Surg 2004; 62: 399-407

[22] Posnick JC. Management of facial fractures in children and adolescents. Ann Plast Surg 1994; 33: 442-457

[23] Imahara SD, Hopper RA, Wang J et al. Patterns and outcomes of pediatric facial fractures in the United States: a survey of the National Trauma Data Bank. J Am Coll Surg 2008; 207: 710716

[24] Horswell BB, Istfan S. Child maltreatment. Oral Maxillofac Surg Clin North Am 2012; 24: 511-517

[25] Mithani SK, St-Hilaire H, Brooke BS et al. Predictable patterns of intracranial and cervical spine injury in craniomaxillofacial trauma: analysis of 4786 patients. Plast Reconstr Surg 2009; 123: 1293-1301

[26] Thorén H, Schaller B, Suominen AL et al. Occurrence and severity of concomitant injuries in other areas than the face in children with mandibular and midfacial fractures. J Oral Maxillofac Surg 2012; 70: 92-96

[27] Hatton MP, Watkins LM, Rubin PA. Orbital fractures in children. Ophthal Plast Reconstr Surg 2001; 17: 174-179

[28] Alcalá-Galiano A, Arribas-García IJ, Martín-Pérez MA et al. Pediatric facial fractures: children are not just small adults. Radiographics 2008; 28: 441-461
[29] Kaban LB. Diagnosis and treatment of fractures of the facial bones in children 1943-1993. J Oral Maxillofac Surg 1993; 51: $722-729$

[30] lizuka T, Thóren H, Annino D] jr. et al. Midfacial fractures in pediatric patients. Frequency, characteristics, and causes. Arch Otolaryngol Head Neck Surg 1995; 121: 1366-1371

[31] Wright RJ, Murakami CS, Ambro BT. Pediatric nasal injuries and management. Facial Plast Surg 2011; 27: 483-490

[32] Yilmaz MS, Guven M, Kabayasoglu G et al. Efficacy of closed reduction for nasal fractures in children. $\mathrm{Br} \mathrm{J}$ Oral Maxillofac Surg 2013; 51: e256-e258

[33] Rohrich RJ, Adams WP. Nasal fracture management: minimizing secondary nasal deformities. Plast Reconstr Surg 2000; 106: $266-273$

[34] Liau JY, Woodlief J, van Aalst JA. Pediatric nasoorbitoethmoid fractures. J Craniofac Surg 2011; 22: 1834-1838

[35] Eppley BL. Use of resorbable plates and screws in pediatric facial fractures. J Oral Maxillofac Surg 2005; 63: 385-391

[36] Hopper RA, Salemy S, Sze RW. Diagnosis of midface fractures with CT: what the surgeon needs to know. Radiographics 2006; 26: 783-793

[37] Sires BS, Stanley RB,Levine LM. Oculocardiac reflex caused by orbital floor trapdoor fracture: an indication for urgent repair. Arch Ophthalmol 1998; 116: 955-956

[38] Ridgway EB, Chen C, Colakoglu S et al. The incidence of lower eyelid malposition after facial fracture repair: a retrospective study and meta-analysis comparing subtarsal, subciliary, and transconjunctival incisions. Plast Reconstr Surg 2009; 124: 1578-1586

[39] Proffit WR, Vig KW, Turvey TA. Early fracture of the mandibular condyles: frequently an unsuspected cause of growth disturbances. Am J Orthod 1980; 78: 1-24

[40] Goth S, Sawatari Y, Peleg M. Management of pediatric mandible fractures. J Craniofac Surg 2012; 23: 47-56

[41] Demianczuk AN, Verchere C, Phillips JH. The effect on facial growth of pediatric mandibular fractures. J Craniofac Surg 1999; 10: 323-328

[42] Smartt JM, Low DW, Bartlett SP. The pediatric mandible: I. A primer on growth and development. Plast Reconstr Surg 2005; 116: 14e-23e

[43] Freudlsperger C, Castrillon-Oberndorfer G, Baechli $\mathrm{H}$ et al. The value of ultrasound-assisted pinned resorbable osteosynthesis for cranial vault remodelling in craniosynostosis. J Craniomaxillofac Surg 2014; 42: 503-507

\section{Bibliografie}

DOI https://doi.org/10.1055/a-0618-1211

OP-JOURNAL 2018; 34: 243-253 @ C Georg Thieme Verlag KG Stuttgart · New York ISSN 0178-1715 